\title{
This Is the Rhythm of Your Eyes: The Phase of Ongoing Electroencephalogram Oscillations Modulates Saccadic Reaction Time
}

\author{
Jan Drewes ${ }^{1,2}$ and Rufin VanRullen ${ }^{1,2}$ \\ ${ }^{1}$ Université de Toulouse, Centre de Recherche Cerveau et Cognition, Université Paul Sabatier, 31062 Toulouse, France, and ${ }^{2}$ Centre National de la Recherche \\ Scientifique, Unité Mixte de Recherche 5549, Faculté de Médecine de Toulouse Rangueil, 31062 Toulouse, France
}

\begin{abstract}
Motor reaction times in humans are highly variable from one trial to the next, even for simple and automatic tasks, such as shifting your gaze to a suddenly appearing target. Although classic models of reaction time generation consider this variability to reflect intrinsic noise, some portion of it could also be attributed to ongoing neuronal processes. For example, variations of alpha rhythm frequency $(8-12 \mathrm{~Hz})$ across individuals, or alpha amplitude across trials, have been related previously to manual reaction time variability. Here we investigate the trial-by-trial influence of oscillatory phase, a dynamic marker of ongoing activity, on saccadic reaction time in three paradigms of increasing cognitive demand (simple reaction time, choice reaction time, and visual discrimination tasks). The phase of ongoing prestimulus activity in the high alpha/low beta range $(11-17 \mathrm{~Hz})$ at frontocentral locations was strongly associated with saccadic response latencies. This relation, present in all three paradigms, peaked for phases recorded $\sim 50 \mathrm{~ms}$ before fixation point offset and $250 \mathrm{~ms}$ before target onset. Reaction times in the most demanding discrimination task fell into two distinct modes reflecting a fast but inaccurate strategy or a slow and efficient one. The phase effect was markedly stronger in the group of subjects using the faster strategy. We conclude that periodic fluctuations of electrical activity attributable to neuronal oscillations can modulate the efficiency of the oculomotor system on a rapid timescale; however, this relation may be obscured when cognitive load also adds a significant contribution to response time variability.
\end{abstract}

\section{Introduction}

A sprint runner may win or lose a race depending solely on their reaction time to the starter gun. Even for such overly practiced activities, the variability in response time (RT) from repetition to repetition cannot be fully removed. Scientists commonly attribute this variability to system noise, as in classic "diffusion" models of reaction time generation (Ratcliff, 1978; Luce, 1986). However, the moment-to-moment variations of ongoing neuronal activity (Buzsaki, 2006) are also likely to contribute to these behavioral variations. Ongoing oscillations are linked to the behavioral state of the person and as such could influence their sensorimotor performance. One extremely rapid source of variability in neuronal activity is the phase of the oscillation: by definition, the phase fluctuates within each oscillatory cycle, whereas the frequency and amplitude are generally modulated over several cycles (Bishop, 1932; Buzsaki and Draguhn, 2004; Lakatos et al., 2005, 2008; Klimesch et al., 2007; Montemurro et al., 2008; Rajkai et al., 2008; Sirota et al., 2008). Here we investigate the possibility that the phase of ongoing oscillatory processes could

Received Sept. 13, 2010; revised Dec. 30, 2010; accepted Jan. 4, 2011.

This research was funded by a European Young Investigators grant and Agence Nationale de la Recherche Grant 06JCJC-0154 (R.V.). We thank N. Busch for his insightful comments.

Correspondence should be addressed to Jan Drewes, CNRS, Centre de Recherche Cerveau et Cognition, UMR5549, Université Paul Sabatier, Toulouse, France. E-mail: Jan.Drewes@cerco.ups-tlse.fr.

DOI:10.1523/JNEUROSCI.4795-10.2011

Copyright $\odot 2011$ the authors $\quad 0270-6474 / 11 / 314698-11 \$ 15.00 / 0$ determine some of the trial-to-trial variability in motor response times within the oculomotor system (i.e., using saccadic responses).

The phase of ongoing oscillations is known to influence cortical excitability (Bishop, 1932; Bartley and Bishop, 1932) and both visual (Jansen and Brandt, 1991) and auditory (Kruglikov and Schiff, 2003) evoked potentials. Recently, the phase of ongoing alpha $(8-13 \mathrm{~Hz})$ and theta $(4-8 \mathrm{~Hz})$ activity was also shown to relate to perceptual (Busch et al., 2009; Mathewson et al., 2009) and attentional (Busch and VanRullen, 2010) performance. Conversely, previous attempts to link the phase of ongoing alpha oscillations with variability in behavioral response times have produced contradictory evidence. Although early reports (Walsh, 1952; O’Hare, 1954) found no significant correlation, later studies (Lansing, 1957; Callaway and Yeager, 1960; Dustman and Beck, 1965) reported weakly significant effects by using restrictive subject and trial selection procedures. A more recent article revealed an influence of prestimulus occipital alpha phase on the probability of producing rapid "express" saccades (Hamm et al., 2010); however, the use of a fixed interstimulus interval in that study precluded a clear distinction between ongoing (i.e., spontaneous) and evoked (i.e., stimulus-locked) oscillations. By and large, the existence of a systematic relation between ongoing oscillatory phase and motor reaction time variability is still an open question.

In the present study, we aim at resolving this question by investigating the specific influence of the phase of ongoing oscil- 

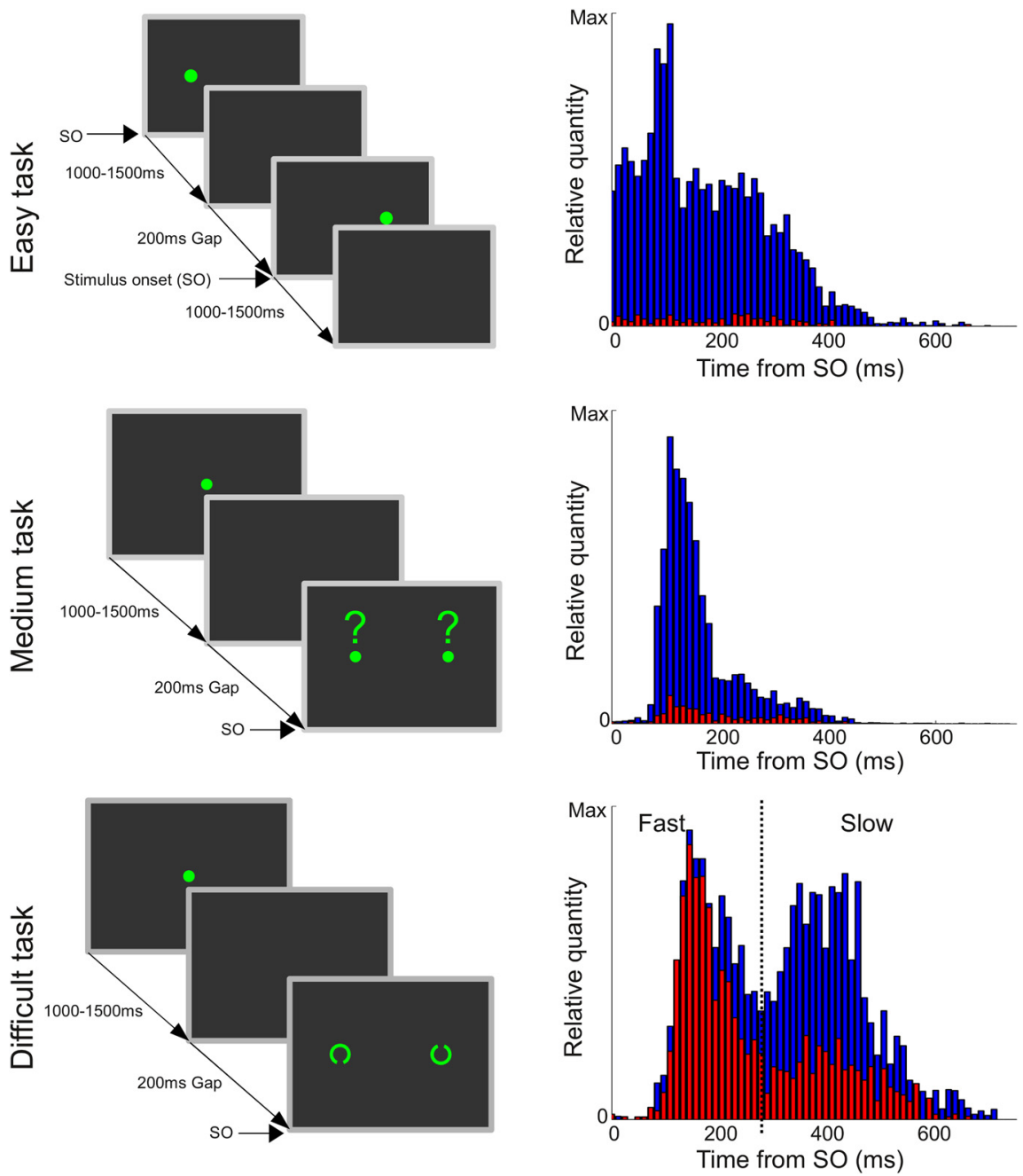

Figure 1. Task design and corresponding RT distributions. Left column, Illustration of the three task paradigms used to record saccadic responses (size not to scale). The easy task involved the detection of the onset of a dot that alternated predictably (but with random intertrial intervals) between the left and right sides of the screen. The medium task required the localization of a single target dot that could appear randomly on either side of the screen. The difficult task implied the discrimination of two simultaneously presented shapes, the target being defined as the one with a gap oriented upward. In all three tasks, participants were instructed to make an eye movement, as quickly and accurately as possible, to the location of the target. Right column, Saccadic RT histograms of all trials, normalized for each subject and then pooled across subjects. The blue bars denote correct trials, and the red bars denote incorrect trials. Note the pronounced bimodality in task 3; the dashed black line at $282 \mathrm{~ms}$ represents the boundary criterion for fast versus slow subjects. S0, Stimulus onset.

latory activity on human saccadic reaction time in three paradigms of increasing cognitive demand. The paradigms were designed to implicate widely different ranges of response times and cognitive strategies, thus maximizing our chances to reveal any systematic relation. We report a strong effect in the high alpha/low beta range $(11-17 \mathrm{~Hz})$, at frontocentral locations, peaking $\sim 50 \mathrm{~ms}$ before fixation point offset and $250 \mathrm{~ms}$ before target onset. The effect was present regardless of cognitive demand but was apparently affected by the subjects' strategy.

\section{Materials and Methods}

\section{Stimulus presentation}

The experiments were programmed in MathWorks Matlab using the Psychophysics Toolbox (Brainard, 1997), on a standard Microsoft Windows computer system. The connected cathode ray tube monitor was set to run with $100 \mathrm{~Hz}$ refresh rate at $1024 \times 768$ pixels resolution. The visual screen area extended $35.6 \times 26.8 \mathrm{~cm}^{2}$ at a viewing distance of $57 \mathrm{~cm}$. The experimental chamber was dimly lit, and care was taken to achieve constant ambient luminance across all subjects and sessions.

\section{Experimental paradigm}

Three saccade production tasks were designed to elicit saccadic responses under increasing cognitive demand (Fig. 1).

Easy task. The first task consisted of a bright green dot $\left(0.2^{\circ}\right.$ visual diameter $)$, which would appear at $8.5^{\circ}$ eccentricity alternately to the left and right of the screen center. After a random interval between 1000 and $1500 \mathrm{~ms}$ (uniform distribution, limited in resolution by the 100 $\mathrm{Hz}$ screen refresh rate), the dot disappeared for a fixed gap duration of $200 \mathrm{~ms}$, after which it reappeared on the opposite side of the screen center at the same eccentricity. At this position, it remained for a random interval between 1000 and $1500 \mathrm{~ms}$, after which the dot disappeared again for a fixed gap duration of $200 \mathrm{~ms}$. The paradigm then looped from the start, creating a continuous, alternating left-right pattern with a random intertrial interval (ITI) of 1200-1700 ms. The participants were instructed to make an eye movement to the position of this dot as fast as possible after its appearance. Although the timing of the interstimulus intervals was randomized, the future position of the stimulus was always predictable; this may induce subjects to produce anticipatory saccades. Subjects were instructed to follow the green dot with their eyes, precisely and as fast as possible, but to avoid anticipation. This task was intended to require a very low cognitive effort; we will thus refer to it as the "easy" condition.

Medium task. The second task started with a central fixation dot of the same size and color as in the first task, which remained on screen for a randomized interval of 1000-1500 ms and then disappeared. After a $200 \mathrm{~ms}$ gap, the dot reappeared randomly $8.5^{\circ}$ to either the left or right of the screen center, where it remained for $1000 \mathrm{~ms}$. The paradigm then looped from the start, resulting in an ITI of $2200-2700 \mathrm{~ms}$. Although fixation timing and target configuration were similar to task 1 , the position of the target stimulus was no longer predictable. The task required a simple decision between the two alternative target locations but, because there was only one target visible at any time, it still allowed for mostly automatic responses (Wurtz and Albano, 1980; Leigh and Zee 1999; Sparks et al., 2000). Subjects were instructed to be as fast and precise as possible. We will refer to this as the "medium" condition.

Difficult task. The final task was identical to the second task until the end of the gap duration; $200 \mathrm{~ms}$ after the gap, two Landolt-style C-rings appeared at an eccentricity of $8.5^{\circ}$, to the left and right of the screen center. Both C-rings measured $0.5^{\circ}$ in diameter and were identical except for their orientation: one of them had a spatial gap pointed upward and one pointed downward. The intertrial timing was unchanged from the medium task. Subjects were instructed to treat only the upward-oriented shape as the target, with the downward-oriented one acting as a distractor. Subjects were again instructed to be as fast and precise as possible. Because of the simultaneous onset and the similarity between target and distractor, this task required some amount of cognitive effort to successfully decide at which of the stimuli to gaze. Within these three tasks, we will therefore refer to this as the "difficult" condition.

All three tasks consisted of the same basic elements: a randomized fixation duration, followed by a $200 \mathrm{~ms}$ gap, and a subsequent target. From fixation onset until just before target onset, all three paradigms can therefore be considered to be equivalent, although the expectations of the 
All trials
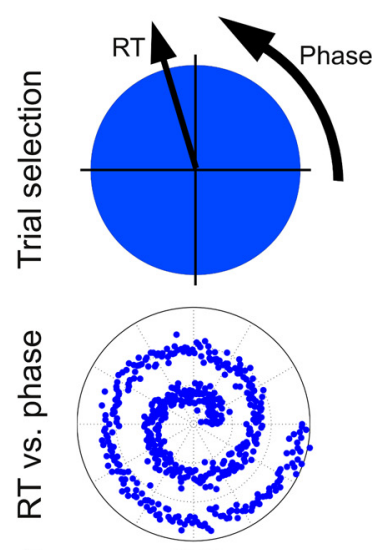

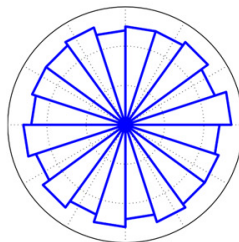

ITC 0

\section{Statistical comparison}
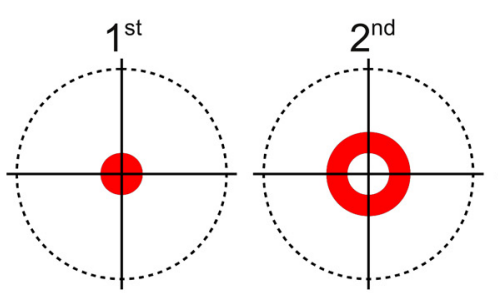

RT quintiles
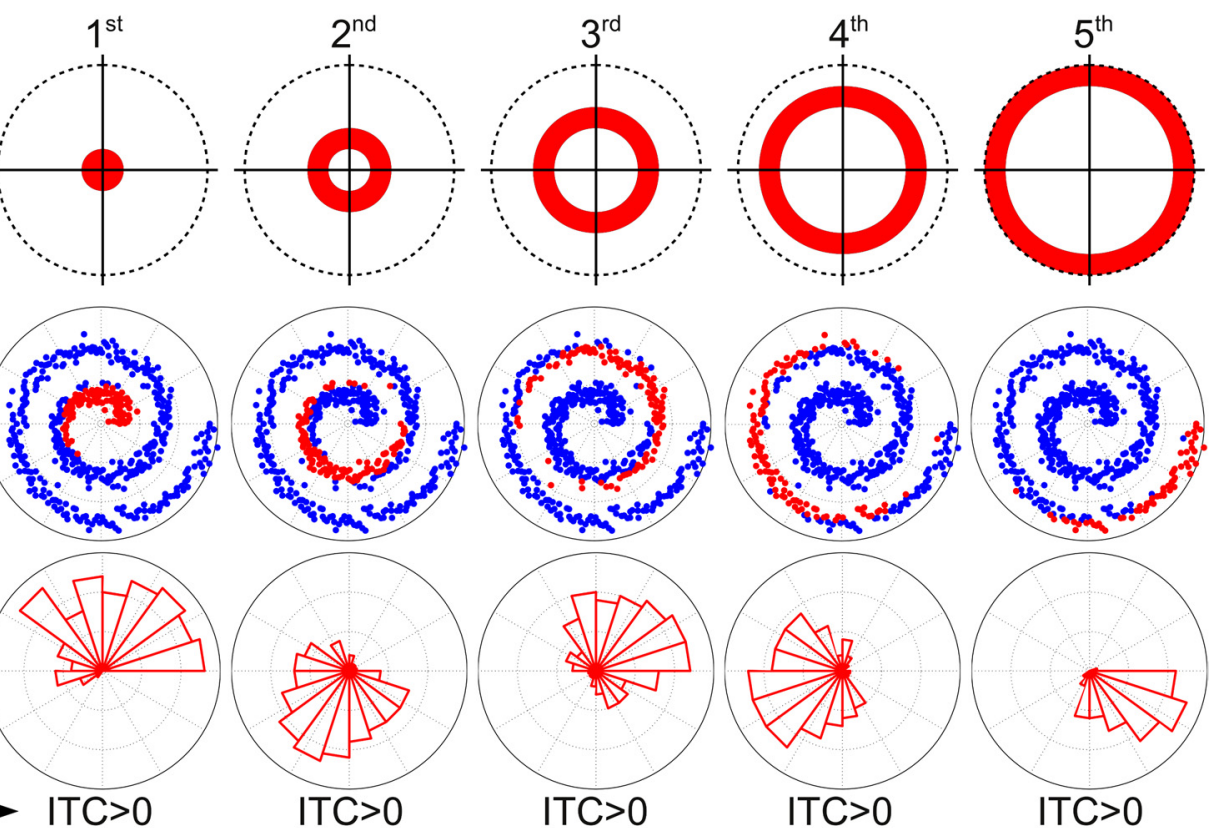

Figure 2. Illustration of our experimental approach with simulated data. Within the first two rows, the phase is represented by the rotational angle and the associated response time by radius (see top left item). The top row illustrates different selections of trial subsets. On the left, a complete selection encompasses all trials. On the right, five successive RT quintiles are shown. For each quintile, the red annulus indicates the range of RTs selected. The middle row displays hypothetical data with a spiral structure: each point corresponds to one trial with a particular phase value (at a given time point and frequency of interest) and an associated RT. The trials selected for each RT quintile are highlighted in red. The bottom row shows the polar phase histograms corresponding to each subset of trials. Phase is represented by the rotational angle and trial number in each bin by the corresponding radius. № apparent phase locking or ITC is expected on the left side when computed across all trials. However, each individual RT quintile shows strong phase locking (right). Within this context, our analysis relied on a statistical comparison between ITC values calculated for the left versus the right histograms. (For statistical consistency, 100 random selections of $20 \%$ of all trials were applied to the left-hand side data, and the resulting ITCs were averaged before comparison with the quintile-specific ITC values on the right).

participating subjects may differ somewhat. Each experimental session was divided into blocks of fixed order, presenting 320 trials of task 1, then 320 trials of task 2, then 200 trials of task 3, in each block. Between two and five blocks were recorded per session, with up to two sessions per subject; all subjects performed a minimum of six blocks. Between-tasks as well as between-blocks subjects were given the opportunity to rest for a short period, if they so desired.

\section{Data recording}

To correlate the eye movements of our subjects with their EEG activity, both eye movements and EEGs were recorded simultaneously. Fifteen subjects participated in the experiment. The age of participants ranged from 23 to 35 years. Seven participants were male; 11 participants were right-handed. A BioSemi EEG system was used to record 64 channels mounted in an elastic cap. Sessions were recorded at a sampling rate of $1024 \mathrm{~Hz}$, which was later downsampled to $256 \mathrm{~Hz}$. Epochs were created ranging from $2000 \mathrm{~ms}$ before to $1000 \mathrm{~ms}$ after stimulus onset. For subjects $1-10$, an optical eye tracker was used to record eye movements (ETL-200; ISCAN). For subjects $11-15$, a three-channel electrooculogram was recorded to determine saccade onset timing and saccade direction. All subjects reported normal or corrected-to-normal vision. The experimental protocol was authorized by the local ethical committee at the Centre National de la Recherche Scientifique.

\section{Analysis}

Preprocessing. EEG data were screened manually on a trial-by-trial basis, rejecting all trials visibly affected by artifacts. Saccade onset times were determined by manual inspection of the horizontal aspect of optical eye traces or electro-oculograms, respectively. Trials were considered valid when subjects did not break fixation and performed a clearly directed saccade at a timing no sooner than the target stimulus onset. Trials containing saccades before stimulus onset were considered to be anticipatory, not stimulus driven, and were discarded. Because all three tasks had two possible target locations (although perfectly predictable in task 1), there was always a chance for decision errors. These were identified by saccades of valid timing yet incorrect initial direction. Correctional saccades potentially occurring at later times were ignored. Of the 15 subjects recorded, one subject was excluded as a result of technical problems during recording, making the alignment of epochs impossible (triggering events were lost). A second subject was excluded because of exceedingly poor compliance with the requirements of the experimental paradigms $(<60 \%$ of the recorded trials were deemed valid because of frequent vertical saccades, extreme anticipations, or long periods of eye closure).

Phase locking. The analysis is aimed at determining the trial-by-trial relationship between prestimulus EEG phases and saccadic RTs.

We hypothesized that saccadic response times are influenced by ongoing EEG oscillations, in such a way that different EEG phases would tend to produce markedly different response latencies. Because these oscillations are ongoing and thus not synchronized to the onset of the stimulus, their distribution over an entire recording session will be randomized and uniform. In other words, no overall phase locking should exist before stimulus onset. However, significant phase locking may be observed if trials are selected within a given range of reaction times. More specifically, our hypothesis predicts that, on a polar plot in which phase is represented by angle and the associated reaction time by radius, the resulting phase-RT mapping should take the form of a spiral (Fig. 2). Note that it is theoretically possible, although not necessary, that the association of latencies and phases spans more than one cycle of the relevant oscillation, resulting in a spiral with several rotations, as illustrated in Figure 2. Whether this is the case or not, when grouping trials by saccadic reaction time (and assuming that a sufficiently narrow range of reaction times is used, in practice spanning less than one spiral rotation), an increase in phase locking ["intertrial coherence" (ITC)] should emerge when comparing with the general ITC across the entire set of trials (Fig. 2).

To summarize, our analysis is based on this simple logic: we compared the phase locking of successive quintiles of the RT distribution (i.e., the 
fastest $20 \%$ for the first quintile, the next $20 \%$ for the second, etc.) to a baseline phase locking computed over the entire RT distribution.

We computed the amount of phase locking or ITC by normalizing the lengths of the complex vectors representing amplitude and phase for all trials and then computing their complex averages according to the following:

$$
\operatorname{ITC}=\left|\frac{1}{n} \sum_{k=1}^{n} e^{i \varphi_{k}}\right|,
$$

where $\varphi$ is the phase angle, and $n$ is the number of trials. The ITC describes the phase locking of a circular distribution of phases by taking values between 0 and 1: 0 when computed on perfectly uniform data, increasing in value with increasing phase synchronization, and reaching 1 for a mono-phase distribution. To derive the necessary phase information, a continuous wavelet transform was computed on single-trial epochs, representing the frequency range from 2 to $100 \mathrm{~Hz}$ in 50 logarithmic steps. The length of the wavelets increased logarithmically from three cycles at $2 \mathrm{~Hz}$ to eight cycles at $100 \mathrm{~Hz}$. For any given time and frequency, the wavelet transform returns complex values representing both phase and amplitude; however, for this analysis, we focused on the phase information only. ITC values were computed for each time-frequency coordinate, creating an intertrial coherence map of the timefrequency plane.

\section{Bootstrapping}

The entirety of the trials collected for a given subject and experimental condition were sorted by reaction time and then subdivided into quintiles, containing disjunctive latency ranges and designed to contain an identical number of trials ( $20 \%$ of the total number) in each quintile. It is important that the range of reaction time selected be as small as possible, to give good temporal resolution and to avoid potential confusion across more than one spiral rotation; simultaneously, the range also needs to be sufficiently wide to provide a sufficient amount of trials for statistical analysis. After exploratory testing, $20 \%$ of the number of valid trials was found to be appropriate.

To compare ITC of a given subject's RT quintiles to the appropriate null distribution, random samples amounting to one-fifth of all the trials of the same subject and condition were drawn and the corresponding ITC was computed. By repeating this process 100 times in a bootstrapping procedure, the mean and SDs for a "null distribution" were computed for each point in the time-frequency plane. The ITC values (and their surrogate mean and SD from the null distribution) were first computed individually for each subject and then combined over subjects, electrodes, and quintiles (and experimental conditions for the analysis described in Fig. 3). The $p$ values were determined by converting the true averaged ITC value into a $t$ value based on the equivalent surrogate distribution mean and SD and then applying a Student's $t$ test for this $t$ value with the corresponding degrees of freedom. The $p$ value indicates the probability that the phase locking computed within the reactiontime-sorted quintiles could be explained under the null hypothesis, i.e., using random trial selection. These $p$ values form a map illustrating at which point in time and frequency the average phase locking of reactiontime-binned trials is greater than that of the general distribution of all trials. To correct for multiple comparisons across time and frequency points, the false discovery rate (FDR) procedure (Benjamini and Hochberg, 1995) was used (setting the expected rate of falsely rejected null hypotheses to 0.001 ).

\section{Methodological differences to previous studies}

Previously published studies relied on strong a priori selection of experimental subjects, oscillation frequency, time point, and electrode location. Probably the most restrictive subject prescreening was done by Lansing (1957), who discarded 92 of 100 tested subjects as a result of insufficient baseline alpha activity. Later, Callaway and Yeager (1960) improved on this but still needed to reject two-thirds of their subjects for similar reasons. Dustman and Beck (1965) used heavy subject prescreening as well but do not give exact numbers. These screenings were intended to select only those subjects with particularly strong alpha range activity to facilitate the analysis. Such a selection may however also raise concerns that any effects discovered might not generalize to a wider experimental population. Our approach required no prescreening of experimental subjects based on oscillatory amplitude, making our conclusions more representative of the general population. Furthermore, these later studies (Callaway and Yeager, 1960; Dustman and Beck, 1965) used online monitoring of EEG activity to trigger the stimulus onset to specific, preselected phases of EEG oscillations. Although this allowed optimizing the number of trials recorded at each of the selected phase offsets, the approach also required the experimenters to limit their frequency of interest to a narrow frequency range, generally centered around $10 \mathrm{~Hz}$; the prestimulus moment and exact scalp location of the phase-triggering function needed to be specified in advance as well. In the discussed studies, only occipital leads were recorded, because the occipital alpha activity was the primary object of interest. In contrast, no a priori frequency or time point selection was necessary for our approach. We were therefore able to detect possible effects in a wide range of frequencies, and, at the same time, our analysis enabled us to detect effects not just precisely at stimulus onset, or at any one fixed time point, but over a range of several hundred milliseconds before the first stimulus event. Even further expanding the coverage of our analysis, the use of current recording equipment enabled us to record from 64 channels distributed across the entire scalp rather than a single preferred topographic region. This allowed us to pick up on possible effects located outside of the occipital area investigated in previous studies.

\section{Results}

\section{Behavior}

The ocular response times in all three tasks were within the range usually reported with similar tasks: $172 \pm 26 \mathrm{~ms}$ for the easy task, $167 \pm 19 \mathrm{~ms}$ for the medium task, and $305 \pm 32 \mathrm{~ms}$ for the difficult task (mean \pm SEM of median RT in correct trials). The histograms for each of the tasks (pooled across subjects) exhibit very distinctive RT distributions (Fig. 1).

In the easy task, a large peak in response quantity can be seen at $\sim 90-120 \mathrm{~ms}$ (peaking at $114 \mathrm{~ms}$ ). Many of the responses occurring before this peak are likely attributable to anticipations, especially because their probability of occurrence is approximately constant down to a RT of 0 ms after stimulus. The percentage correct was very high in the easy task, with an average $96.2 \pm 1.4 \%$ correct. Because the locations of upcoming targets in this task were perfectly predictable, any errors may be explained by lapses of attention or occasional failures of the ocular position recording system.

In the medium task, a large and sharply defined peak (maximum bin centered at $114 \mathrm{~ms}$ ) dominates the histogram. Fewer anticipations were observed in this task. This sharp and unimodal distribution corresponds well to a mostly "automatic" mechanism. Our subjects delivered a high level of accuracy, with an average $92.8 \pm 2.0 \%$ correct.

The difficult task produced a bimodal RT histogram. The first modality showed a clear first peak at $150 \mathrm{~ms}$ (center of maximum bin), and the second modality featured a less well defined maximum between 350 and $460 \mathrm{~ms}$. In this task, the accuracy was less than in the previous tasks, with an average of $66.0 \pm 3.6 \%$ correct. The difficult task was designed to be of comparatively high cognitive demand, with a high degree of similarity between target and distractor stimuli. Thus, the larger number of error trials may be attributable to genuine errors in judgment. The two modes in the RT histogram reflect two different strategies used by different subjects: although faster, the responses in the first mode are inaccurate, close to chance performance, and the accuracy of the trials in the second, slower mode is much higher, at $57.6 \%$ correct for trials faster than $282 \mathrm{~ms}$ and $73.3 \%$ correct for slower trials (boundary set at the minimum between peaks) (Fig. 1). As we will 
see later, individual subjects tended to apply one response strategy or the other rather than alternate between the two strategies.

\section{Phase-RT relations}

Our main hypothesis is that the phase of ongoing oscillations constrains the range of RTs that can be produced on a given trial. A systematic relationship between phase and RT would take the form of a spiral in a polar plot representing RT as a function of phase angle (Fig. 2). Note that the spiral may contain one (if the phase-RT relation is bijective or "one-toone") or possibly several rotations (if the RT distribution spans several cycles of the ongoing oscillation). In either case, a global measure of ITC across all trials would reveal only minimal phase locking (indeed, the randomized intertrial intervals prevent ongoing oscillations to have any consistent temporal relationship with stimulus onset). However, by grouping trials into sufficiently small RT bins, the relationship should be revealed as an increase in phase locking compared with a random trial selection (Fig. 2). Thus, our analysis relies on a statistical comparison (bootstrapping) between ITC computed over separate RT quintiles and ITC computed over the same number of trials drawn randomly from all RT quintiles (representing the ITC distribution under the null hypothesis).

\section{Overview}

As a first step, the collected data was pooled across all conditions and electrodes (Fig. 3). The bootstrapping procedure revealed an area of significantly higher $\left(p<10^{-8}\right)$ phase locking when computed over RT-sorted quintiles than over random trial samples, at $47 \mathrm{~ms}$ before the gap onset (the disappearance of the fixation point), i.e., $247 \mathrm{~ms}$ before stimulus onset, peaking at a frequency of $13.6 \mathrm{~Hz}$. This effect remained significant after correction for multiple comparisons using the FDR procedure. Because the interval between trial onset and gap onset was randomized and unpredictable on each trial, the fact that this effect occurred before the gap onset is a clear indication that it was produced by ongoing rather than stimulus-induced oscillations. In fact, the precise temporal position of this effect might reflect the last truly ongoing cycle before the gap onset: a transient in the stimulation that can create an evoked potential and reset ongoing oscillations. By mapping the computed $p$ values contained in the region of interest onto a scalp-electrode layout (Fig. 3B), we found this effect to be attributed primarily to oscillatory activity over a frontocentral region, with possibly a minor influence from occipital areas. To improve the signal-to-noise ratio, all additional analyses were based on the selection of 12 frontocentral electrodes outlined in Figure $3 B$.

The same bootstrapping analysis also revealed a second, rather large region of the time-frequency plane in which phase locking was highly significant $\left(p<10^{-8}\right)$ : this effect can be seen starting just after the gap onset at low frequencies and culminating in a strong peak $\sim 100-150 \mathrm{~ms}$ after stimulus onset, ranging in frequency from the lower limit of our analysis (2 Hz) up to $\sim 60 \mathrm{~Hz}$
(Fig. 3A). This massive effect is most likely either a reflection of saccadic programming mechanisms in the brain or contamination from eye movements, starting after stimulus-locked events (the gap) and reaching a peak just before the time of occurrence of the majority of saccades. This interpretation is supported by the topography presented in Figure $3 C$, where it is apparent that post-gap effects are maximal over fronto-ocular electrodes. During the sequence of sensory processing, response preparation, and execution, large portions of the visual and oculomotor systems are likely to be activated at a more or less fixed time preceding (or following) the saccadic response. These poststimulus processes would show increased phase locking when grouped with respect to the moment of the saccade, as done in our analysis. Even the earliest part of this significant time-frequency region, between gap and stimulus onset, could be explained by the temporal variability of sensorimotor processes occurring in response to the gap (which has a fixed, fully predictable temporal relation to the moment of stimulus onset). In other words, no part of this secondary effect found after the gap onset can truly be said to reflect the influence of ongoing rather than stimulusinduced oscillations.

\section{Effects of task difficulty}

Next, we applied the same analysis technique separately to the three individual tasks, using only the fronto-central electrode region of interest determined above. For all three tasks, significant pre-gap effects $\left(p<10^{-6}\right)$ were found in the same approximate time-frequency location as the original effect reported above (Fig. 4).

The peak happened at slightly different time-frequency points in the three tasks: for the easy task, the effect occurred just before the gap onset $(25 \mathrm{~ms})$ and peaked at $14.5 \mathrm{~Hz}$, for the intermediate task the center was located at $\sim 50 \mathrm{~ms}$ before the gap onset and 14 $\mathrm{Hz}$, and in the difficult task the duration of the effect was somewhat elongated but had a peak at $\sim 90 \mathrm{~ms}$ before the gap onset and $12.5 \mathrm{~Hz}$. 

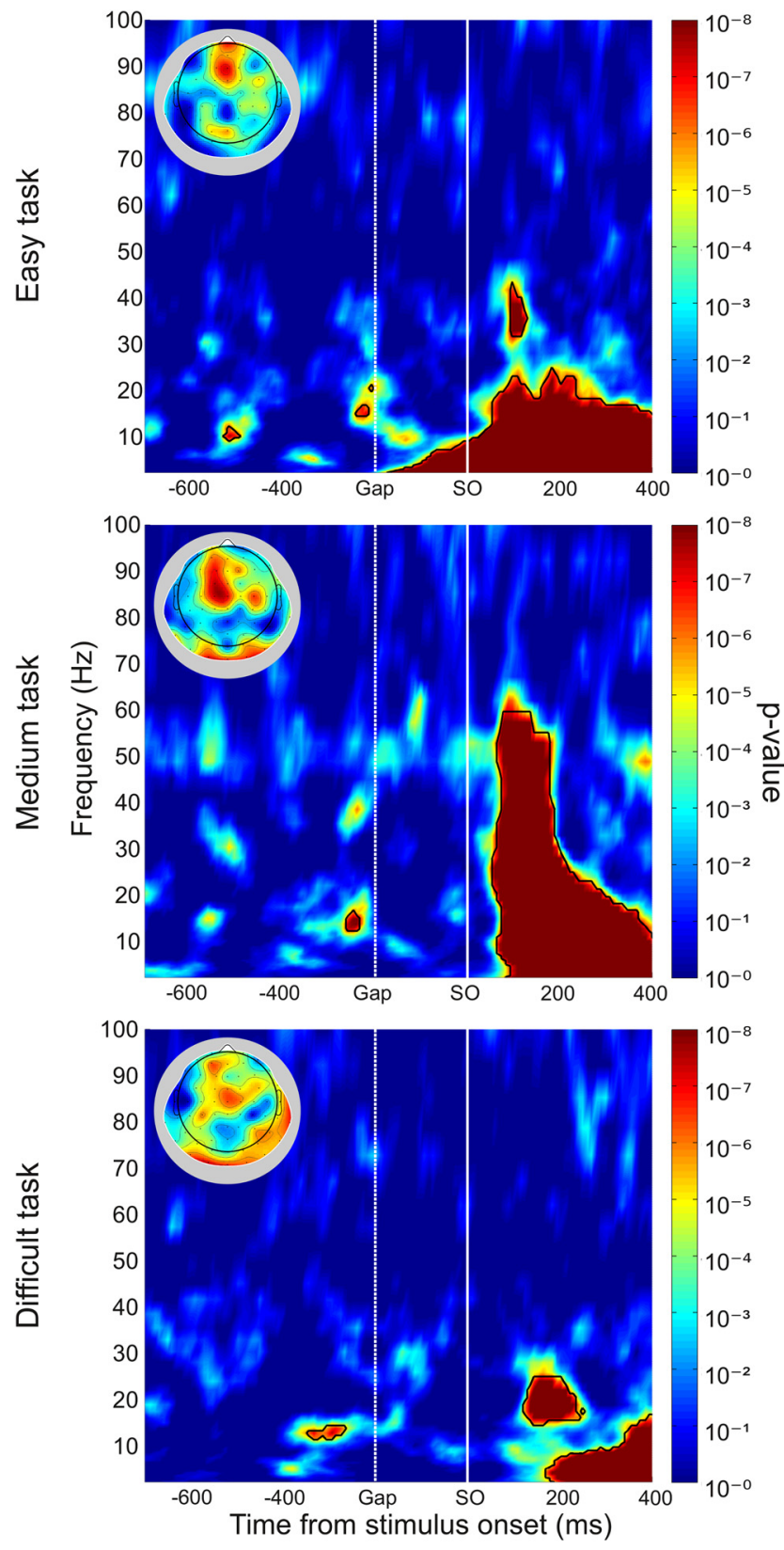

Figure 4. Results for the three individual tasks. p values resulting from the bootstrapping ITC analysis (as in Fig. 3A) are shown separately for each of the three tasks. In all three tasks, significant effects can be seen within a similar time-frequency region (11-17 Hz, 0-100 ms before gap onset). Black outlines highlight regions above FDR threshold. Small insets show topography of activity in the region of interest defined in Figure 3B. SO, Stimulus onset.

In the easy task, a second effect exceeded the FDR threshold, located at $\sim 10 \mathrm{~Hz}$ and $300 \mathrm{~ms}$ before the gap onset (i.e., $500 \mathrm{~ms}$ before stimulus onset). In this task, the upcoming target location was always known in advance; although the fixation duration was randomized, the following gap interval was fixed, and, therefore, from the moment of the gap onset, both target onset timing and target location were predictable. The fixation offset/gap onset may therefore have served as an alternate "go" signal, triggering early responses. The very early prestimulus effect found in this task, occurring $\sim 300 \mathrm{~ms}$ before the gap onset, may thus be attributed to those trials in which the gap onset rather than the stimulus onset was used as the go signal. In turn, these trials may correspond to the numerous "anticipations" (with reaction times close to zero) recorded in this task. In the medium task, the future target location was unpredictable, preventing the use of the fixation offset as an alternative response trigger.

Finally, note that poststimulus (or post-gap) effects are present in all three tasks as observed previously, albeit in somewhat different regions of the time-frequency plane. Exclusively in the easy task, the poststimulus effect starts just at the beginning of the gap at the lowest frequency $(\sim 2 \mathrm{~Hz})$ and progressively involves higher frequencies (up to $\sim 17 \mathrm{~Hz}$ ) until stimulus onset; after stimulus onset, this effect continues to grow in frequency and combines with the massive saccadic effect also observed in the other two tasks. The earlier effect in the easy task is likely attributable to the large number of saccadic anticipations in this task, as manifested in the very large spread of RTs illustrated in Figure 2 (top right); this increased RT variability will result in a widening of the phase-RT effect at lower frequencies as a result of their tolerance for moderate temporal misalignments. Because it is not possible to assess whether post-gap effects are attributable to ongoing rather than stimulus-induced oscillations, we did not explore these findings in more detail.

\section{Fast versus slow subjects}

The results described so far suggest that the influence of $\sim 13 \mathrm{~Hz}$ ongoing oscillations on saccadic RT does not depend on task difficulty or cognitive demand. To probe this finding further, we focused on the difficult task, which displayed a pronounced bimodality in its RT distribution pooled across subjects (Fig. 1), suggestive of the existence of two response strategies: a rapid but poorly accurate one, and a slow and more precise one. Interestingly, the corresponding histograms for most of our individual subjects ( 12 of 13) were in fact unimodal in nature, indicating the preferential use of only one of these two strategies. We selected the minimum of the valley between the two peaks in the pooled across-subjects histogram as a boundary (282 ms) (Fig. 1) and sorted our subjects according to their individual preferred modes. Subjects with median latencies faster than this limit were classified as the "fast" group ( $n=6$; median RT, $197 \pm 17 \mathrm{~ms})$ and those with median latencies slower than the boundary as the "slow" group ( $n=6$; median RT, $421 \pm 14 \mathrm{~ms})$. One subject (S13) was excluded from this analysis because of a pronounced RT bimodality that did not allow for a clear group assignment. The group-average RT histograms are shown in Figure 5, $A$ and $B$. The speed-accuracy tradeoff between strategies is obvious in the fact that by grouping our subjects into fast and slow latencies, we also grouped them into "inaccurate" (fast group; median accuracy, $57.8 \pm 1.7 \%$ correct) and "accurate" (slow group; median accuracy, $76.3 \pm 6.3 \%$ correct) behavior. When computing the time-frequency bootstrapping analysis separately for these two subject groups, we found a very strong effect $\left(p<10^{-11}\right)$ within the fast/inaccurate group of subjects, at a time-frequency location (peaking at $12.5 \mathrm{~Hz}$ and $100 \mathrm{~ms}$ before gap onset) similar to the primary effect found previously (Fig. $5 C$ ). A small portion of this effect is still visible $\left(p<10^{-5}\right)$ when looking at the slow/ accurate group of subjects (Fig. 5D), but it does not exceed the FDR threshold. This weak effect may be explained by a small amount of fast saccades made by the subjects assigned to the slow group (Fig. 5B). We also computed a direct statistical comparison between phase-locking values obtained for the two groups of subjects and found a statistically significant $(p<0.0001)$ difference within the time-frequency region of interest (data not shown).

In conclusion, the difficult task was apparently treated by subjects according to two possible strategies. The first one consisted 
in providing response times similar to those obtained in the medium task, at the cost of decreased accuracy; it may be assumed that these subjects applied the same strategy as used in the medium task. The second one consisted of slowing down saccadic responses to maintain accuracy at a reasonable level. Interestingly, only the first strategy seemed to yield a significant phase-RT relation. Within the second subject group, the additional cognitive processing (or simply the noise occurring during the extended duration of the stimulus-response cycle) may have added another source of variance to the RT generation process.

\section{Phase progression}

Our previous findings clearly support the idea of a relation between the phase of ongoing $\sim 13 \mathrm{~Hz}$ oscillations and the associated ocular response RT. However, our analysis does not allow us to draw conclusions about the specific nature of this relation: it could very well be that only one or two of the five RT quintiles is really associated with particular prestimulus phase values, with the rest of the RT histogram being more uniformly distributed in terms of phase. Conversely, there could be a steadily high amount of phase locking with a systematic shift of the preferred phase value when RT increases, as suggested by the "spiral" illustrated in Figure 2. Furthermore, the phase locking was calculated independently for the quintiles of each subject, before these phase locking values were averaged over subjects. Thus, our result of a significantly high phase locking could be attributable to a systematic tendency in all observers for specific phases to be associated with faster or slower RTs or it could be attributed to an idiosyncratic relation between phase and RT that would vary across subjects. The subsequent analysis was designed to simultaneously address these two issues.

We pooled all trials $(n=14,070)$ from all conditions across all subjects and assessed the relationship between RT and the phase of ongoing oscillatory activity recorded on the same trial at 13.6 $\mathrm{Hz}$ and $47 \mathrm{~ms}$ before gap onset. A window containing $20 \%$ of all trials (comparable with one quintile of an imaginary "global" subject) was moved progressively across the entire RT distribution (Fig. 6A), starting with the first possible quintile (20\% fastest trials across all subjects), shifting by steps of one trial until reaching the last possible quintile (20\% slowest trials across all subjects). At each step, a Von Mises circular distribution was fitted to the phase histogram of trials falling into the sliding window (Berens, 2009). This distribution is characterized by an optimal phase angle, as well as a concentration parameter representing the amount of phase locking. The optimal phase angle and the concentration parameter obtained at each step are represented in graphical format in Figure $6 B$, together with the corresponding significance thresholds. Snapshots of the phase histogram and the corresponding Von Mises fit, taken at times corresponding to the five non-overlapping quintiles, can be seen in Figure 6C. Rayleigh tests computed on the estimated Von Mises distributions of each snapshot confirmed that the general distribution of phases in all of the five snapshots diverged significantly from randomness
(Fig. 6C). An animated version of Figure 6 is provided as supplemental material (see Notes).

After a few steps of relative constancy, the optimal phase within the sliding trial window was found to progress continuously with increasing RT, until the moving window reached the approximate position of the fourth quintile. Until this step, the concentration parameter virtually always remained above the $p<0.01$ threshold and above the $p<0.001$ threshold for $\sim 80 \%$ of the steps. After passing the position of the fourth quintile, the concentration parameter declined for a few steps to belowsignificance levels $(p>0.05)$, during which the optimal phase slipped forward by slightly $>180^{\circ}$. Although the concentration parameter returned to significant levels after this $(p<0.01)$, the optimal phase stayed mostly constant for the remaining RT range. The overall relation between median $\mathrm{RT}$ and optimal phase in the sliding trial window is illustrated by the polar plot inserted in Figure $6 \mathrm{~B}$, which takes the form of the expected spiral.

We conclude that the optimal phase of ongoing $~ 13 \mathrm{~Hz}$ EEG oscillations advances reasonably continuously with increasing response latencies and that this relation holds significantly for most of the RT windows considered, not just for one or two quintiles. In addition, the existence of such a relation between phase and RT after having pooled trials from all participants indicates that the relationship must be fairly consistent across subjects: similar phases of the ongoing oscillation tend to induce comparably fast reaction times.

\section{Discussion}

In three tasks of increasing cognitive demand, we investigated the relationship between the phase of ongoing electroencephalographic activity at or before the time of visual stimulation and the associated human ocular response times. We found a highly significant relation between RTs and prestimulus oscillatory phase 


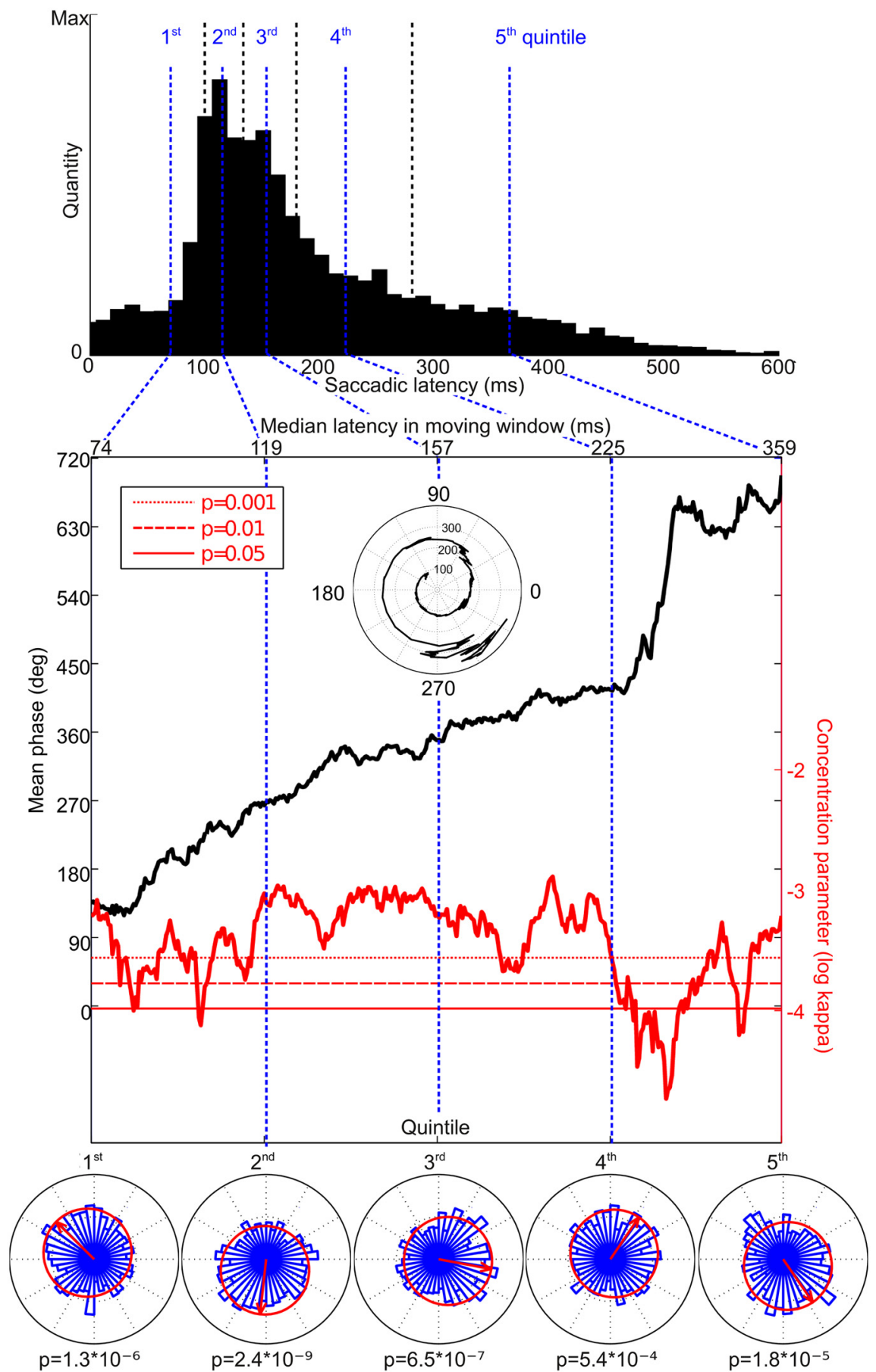

Figure 6. Global phase progression. Top, Latency histogram across all subjects, experimental tasks, and trials. The vertical black dashed lines delimit quintiles, and the blue dashed lines traversing the top and middle graphs mark the median RT of the respective quintiles. A sliding window containing $20 \%$ of trials was moved progressively across the RT histogram, starting with the first quintile, shifting at each step by one trial until reaching the last quintile. Middle graph, Phase progression in moving window. The black line represents the mean phase in the subset of trials (unwrapped for increased readability), and the red line represents the concentration parameter (both mean phase and concentration parameters were derived using a Von Mises fit). Three red horizontal lines specify equivalent $p$ levels of the concentration parameter of $p<0.05, p<0.01$, and $p<0.001$. The inset illustrates this phase progression in polar coordinates (phase represented by angle and RT by radius): a spiral shape emerges. Bottom row, Phase histograms of all trials in the moving window, at time points corresponding to the five disjoint quintiles used in previous analyses. The red arrow indicates the mean phase angle, and the red circle illustrates the corresponding Von Mises fit. Results of Rayleigh tests of the significance of the mean direction in a circular distribution are reported below. For increased visibility (and for display purposes only), a fixed trial number was subtracted from each phase bin.

effect was most pronounced in fast, inaccurate responses and less in slow, accurate ones, suggesting that more cognitive responses may be less affected by the current phase of the ongoing activity (Fig. 5). When pooled across all tasks and subjects, the mean phase at the optimal time-frequency point continuously progressed with increasing RT (Fig. 6), suggesting a consistent relationship between the phase of ongoing activity and the corresponding reaction time.

The notion that ongoing neuronal activity can influence reaction times has been examined in the past. The amplitude of ongoing prestimulus activity, particularly in the alpha band $(8-13 \mathrm{~Hz})$, is known to be related to fluctuations in the response of the visual system (e.g., visual evoked potentials) (Jansen and Brandt, 1991) and in attentional and perceptual performance (Ergenoglu et al., 2004; Hanslmayr et al., 2005, 2007; Thut et al., 2006; van Dijk et al., 2008). Thus, not surprisingly, changes in alpha-band oscillatory amplitude have also been linked to reaction time differences (Ota et al., 1996; Linkenkaer-Hansen et al., 2004; Bollimunta et al., 2008; Kirschfeld, 2008). In this paper, we focused on more dynamic signatures of ongoing activity, such as those carried by phase rather than amplitude information.

Under certain circumstances, the histogram of manual, vocal, or saccadic RTs to a visual stimulus can show a number of peaks and troughs, with regular spacing between them (Venables, 1960; Latour, 1967; White and Harter, 1969; Treisman et al., 1990; Dehaene, 1993). This kind of periodicity is indeed suggestive of a rapid periodic influence on the RT generation process, yet in these paradigms, the stimulus onset is unpredictable and bears no systematic relation with ongoing internal processes. Thus, periodicities visible in RT histograms must reflect either the phase reset of an internal rhythm or an additional stimulus-evoked oscillation (Dehaene, 1993); they do not, however, indicate a direct relation between ongoing oscillatory activity and response time variability.

Surwillo (1963a,b, 1964) very convincingly reported that the dominant alpha period of each subject is positively correlated with their average manual response latency to an auditory stimulus. Although the EEG periodicity was determined after stimulus and was thus affected by evoked

(Fig. 3), with an overall peak at $13.6 \mathrm{~Hz}(11-17 \mathrm{~Hz})$ and $\sim 50 \mathrm{~ms}$ before the first stimulus event (the gap onset). This main effect was found across all three tasks, albeit with small variations in timing and frequency (Fig. 4). In a demanding decision task, the in addition to ongoing activity, this result was taken to imply that ongoing EEG oscillatory cycles could function as a clocking mechanism for programming motor responses. Along this line of reasoning, the specific phase of the ongoing EEG cycle at which a 
stimulus is presented on a given trial should affect the corresponding response time. However, experimental evidence directly relating single-trial ongoing oscillatory phase and reaction time so far had been sparse and mitigated (Walsh, 1952; O'Hare, 1954; Callaway and Yeager, 1960; Venables, 1960; Surwillo, 1963a,b, 1964; Dustman and Beck, 1965). We believe that many factors could have contributed to the success of the present study.

First, with the exception of Venables (1960) who used vocal responses, all of the above-mentioned work used manual responses [button press (Callaway and Yeager, 1960) or motor tremor (Lansing, 1957; Dustman and Beck, 1965)] to measure response times. Generally, the longer the average reaction time, the more difficult it will be to reliably show a phase correlation, because the phase of any cyclic brain activity will tend to diverge over time as a result of noise. Therefore, to obtain optimal phase coherence within any stimulus-response cycle, as fast a reaction time as possible is desirable. Eye movements in response to visual stimulation are among the fastest voluntary reaction times known in humans, significantly faster than manual responses. The use of ocular response times therefore provided us with a means to shorten the input-output loop and thus may have allowed for a more direct and coherent way of detecting phase correlations between ongoing activity and response times. (This idea may also explain why the phase-RT relation was less visible in slow subjects during the difficult task: these subjects produced RTs on average $200 \mathrm{~ms}$ longer than the fast subjects.) Note that Gaarder et al. (1966) reported significant phase locking between the ongoing alpha rhythm and the spontaneous occurrence of microsaccades during fixation; however, the present study is the first to report a direct link between ongoing oscillatory phase and the reaction time of a voluntary oculomotor response.

Second, previously published studies relied on strong a priori selection of experimental subjects, oscillation frequency, time point, and electrode location. No such preselection was necessary in our approach.

Third, and perhaps most importantly, our analysis considered the possibility that a phase-to-reaction time mapping may involve several consecutive oscillatory cycles rather than a simple one-to-one mapping (Fig. 2). Previous approaches assumed a more or less bijective relation between the phase of ongoing oscillations and resulting RTs, with one particular phase resulting in one particular expected reaction time. Hence, they grouped trials by oscillatory phase and searched for RT differences between these trial groups. This logic should imply that the variability in RT that can possibly be accounted for by single-trial ongoing phase is limited to one period of the relevant oscillation, an assumption generally not supported by the width of the RT distribution, which frequently spans the duration of several oscillatory periods. As an example, the difference in median RT between the first and fifth quintile of the reaction time distribution recorded for fast subjects in Figure $5 A$ is $149 \pm 12 \mathrm{~ms}$, whereas the peak frequency of the ongoing oscillation that best accounts for this variability is $12.5 \mathrm{~Hz}$, corresponding to a $80 \mathrm{~ms}$ period. That RT variability encompasses more than one ongoing oscillatory cycle is also apparent in the spiral shape summarizing our experimental data in Figure 6. If the number of oscillatory cycles that contribute to RT generation exceeds one, a given phase angle may be associated with more than one preferred RT range, effectively dooming any analysis based on grouping by prestimulus phase. Conversely, when grouping trials by sufficiently narrow ranges of saccadic $\mathrm{RT}$, no assumption needs to be made about the number of consecutive oscillatory cycles involved in the forming of the RT distribution. Indeed, this novel analysis revealed that a specific prestimulus phase value tended to be associated with each saccadic RT group, but the full range of phase values spanned more than one oscillatory cycle (Fig. 6).

A phase-RT relation spanning multiple cycles could happen, for example, as a consequence of the temporal variability of the sensory information about a newly appearing stimulus: on some trials, this information may arrive at an "inopportune" time, causing a delay in the system before the information is then processed in a subsequent cycle. Alternatively, sensory information may need to accumulate until a threshold is reached before additional processing takes place; again, the moment at which the threshold is reached would determine whether the corresponding information is processed in a given cycle or a following one. One benefit of this scheme would be that a new processing cycle is only initiated once the to-be-processed information has reached a sufficient magnitude to justify the energy expense. Regardless of the underlying neuronal origin, our findings imply that the precise prestimulus phase determines not one particular instant of a unique response cycle at which the reaction time could be initiated but rather a number of successive "temporal windows of opportunity" for reaction time generation, recurring at a specific frequency.

The principal location of the phase-RT relationship was observed over frontocentral electrodes (Fig. 3). Of course, EEG topographies cannot be directly interpreted in terms of the underlying sources; additionally, these results represent the location of highest statistical significance, which is not necessarily identical to the physiological origin of the relevant oscillatory activity. Nevertheless, if we are willing to consider the frontocentral topography as indicative of a frontocentral cortical origin, the frontal eye fields (FEFs) may seem to be a logical candidate as the source of the effect. Although the superior colliculi are generally believed to control reflexive saccades and bottom-up gaze shifts (Leigh and Zee, 1999), the FEFs are considered to be responsible for more voluntary changes in gaze and for the top-down inhibition of reflexive saccades (Neggers et al., 2005). For example, they are involved in visual search tasks in which multiple stimuli are simultaneously present and a decision is required to shift gaze onto the target (Schall and Thompson, 1999; Sato et al., 2001). Aside from the easy task in which target location and timing (after gap onset) were entirely predictable and in which we recorded a large number of anticipations (possibly indicative of involuntary responses mediated by the superior colliculus), the medium and difficult tasks both required such a decision between two equiprobable alternatives, justifying a potential involvement of the FEF. Conversely, the effect was markedly weaker within the group of slow subjects in the latter task; as mentioned previously, this may reflect the contribution of additional areas (e.g., parietal) (Gottlieb et al., 1998) to overcome the challenging perceptual decision, or it may simply be the consequence of diminishing statistical significance (because of phase divergence over time) with increasing duration of the stimulus-response cycle. Finally, the scalp topographies are also compatible with another possible source: the anterior cingulate cortex, known to play an important role in the monitoring of ongoing cognitive processes and the adjustment of behavioral responses (Pastötter et al., 2010).

Recently, Busch et al. (2009) reported that single-trial prestimulus phase at $\sim 7 \mathrm{~Hz}$ in a very similar frontocentral topographic region predicts subsequent visual perception of a threshold stimulus (Busch et al., 2009). Busch and VanRullen (2010) later found this effect to be mediated by $\sim 7 \mathrm{~Hz}$ periodic fluctuations of sustained attention. In this context, the influence of prestimulus phase on reaction time found here may be indic- 
ative of analogous attentional fluctuations taking place in our saccadic tasks. However, the frequency of this periodic influence in our case was approximately twice that reported previously. Whether this difference reflects harmonic variants of a single periodic mechanism or the operation of two distinct mechanisms altogether will have to be decided on the basis of future research.

\section{Conclusions}

The single-trial phase of ongoing oscillations has been shown recently to predict near-threshold visual perception (Busch et al., 2009), attentional performance (Busch and VanRullen, 2010), and meta-contrast masking efficiency (Mathewson et al., 2009). It has been postulated for decades that a correlation with response times also exists, but only weakly significant results had been reported previously. The results presented here should eliminate any doubt that the phase of ongoing oscillations does in fact have an important influence on the continuous measure of human ocular response latencies. This may be compatible with the idea of a cyclic nature of sensory processing, in line with the original concept of a "perceptual moment" (Stroud, 1956, 1967; Varela et al., 1981; Purves et al., 1996; VanRullen and Koch, 2003).

\section{Notes}

An animated version of Figure 6 is provided at http://www.cerco.ups-tlse. fr/ $\sim$ rufin/phase_RT.html.

\section{References}

Bartley SH, Bishop GH (1932) The cortical response to stimulation of the optic nerve in the rabbit. Am J Physiol 103:159-172.

Benjamini Y, Hochberg Y (1995) Controlling the false discovery rate: a practical and powerful approach to multiple testing. J R Stat Soc Series B 57:289-300.

Berens P (2009) CircStat: a Matlab Toolbox for circular statistics. J Stat Softw 31:1-21.

Bishop GH (1932) Cyclic changes in excitability of the optic pathway of the rabbit. Am J Physiol 103:213-224.

Bollimunta A, Chen Y, Schroeder CE, Ding M (2008) Neuronal mechanisms of cortical alpha oscillations in awake-behaving macaques. J Neurosci 28:9976-9988.

Brainard DH (1997) The Psychophysics Toolbox. Spat Vis 10:433-436.

Busch NA, Dubois J, VanRullen R (2009) The phase of ongoing EEG oscillations predicts visual perception. J Neurosci 29:7869-7876.

Busch NA, VanRullen R (2010) Spontaneous EEG oscillations reveal periodic sampling of visual attention. Proc Natl Acad Sci USA 107:16048-16053.

Buzsaki G (2006) Rhythms of the brain, Ed 1. Oxford, UK: Oxford UP.

Buzsáki G, Draguhn A (2004) Neuronal oscillations in cortical networks. Science 304:1926-1929.

Callaway E 3rd, Yeager CL (1960) Relationship between reaction time and electroencephalographic alpha phase. Science 132:1765-1766.

Dehaene S (1993) Temporal oscillations in human perception. Psychol Sci $4: 264-270$.

Dustman RE, Beck EC (1965) Phase of alpha brain waves, reaction time and visually evoked potentials. Electroenceph Clin Neurophysiol 18:433-440.

Ergenoglu T, Demiralp T, Bayraktaroglu Z, Ergen M, Beydagi H, Uresin Y (2004) Alpha rhythm of the EEG modulates visual detection performance in humans. Brain Res Cogn Brain Res 20:376-383.

Gaarder K, Koresko R, Kropfl W (1966) The phasic relation of a component of alpha rhythm to fixation saccadic eye movements. Electroencephalogr Clin Neurophysiol 21:544-551.

Gottlieb JP, Kusunoki M, Goldberg ME (1998) The representation of visual salience in monkey parietal cortex. Nature 391:481-484.

Hamm JP, Dyckman KA, Ethridge LE, McDowell JE, Clementz BA (2010) Preparatory activations across a distributed cortical network determine production of express saccades in humans. J Neurosci 30:7350-7357.

Hanslmayr S, Klimesch W, Sauseng P, Gruber W, Doppelmayr M, Freunberger R, Pecherstorfer T (2005) Visual discrimination performance is related to decreased alpha amplitude but increased phase locking. Neurosci Lett 375:64-68.

Hanslmayr S, Aslan A, Staudigl T, Klimesch W, Herrmann CS, Bäuml KH (2007) Prestimulus oscillations predict visual perception performance between and within subjects. Neuroimage 37:1465-1473.

Jansen BH, Brandt ME (1991) The effect of the phase of prestimulus alpha activity on the averaged visual evoked response. Electroencephalogr Clin Neurophysiol 80:241-250.

Kirschfeld K (2008) Relationship between the amplitude of alpha waves and reaction time. Neuroreport 19:907-910.

Klimesch W, Sauseng P, Hanslmayr S (2007) EEG alpha oscillations: the inhibition-timing hypothesis. Brain Res Rev 53:63-88.

Kruglikov SY, Schiff SJ (2003) Interplay of electroencephalogram phase and auditory-evoked neural activity. J Neurosci 23:10122-10127.

Lakatos P, Shah AS, Knuth KH, Ulbert I, Karmos G, Schroeder CE (2005) An oscillatory hierarchy controlling neuronal excitability and stimulus processing in the auditory cortex. J Neurophysiol 94:1904-1911.

Lakatos P, Karmos G, Mehta AD, Ulbert I, Schroeder CE (2008) Entrainment of neuronal oscillations as a mechanism of attentional selection. Science 320:110-113.

Lansing RW (1957) Relation of brain and tremor rhythms to visual reaction time. Electroencephalogr Clin Neurophysiol 9:497-504.

Latour PL (1967) Evidence of internal clocks in the human operator. Acta Psychologica 27:341-348.

Leigh RJ, Zee DS (1999) The neurology of eye movements, Ed 3. Oxford, UK: Oxford UP.

Linkenkaer-Hansen K, Nikulin VV, Palva S, Ilmoniemi RJ, Palva JM (2004) Prestimulus oscillations enhance psychophysical performance in humans. J Neurosci 24:10186-10190.

Luce RD (1986) Response times: their role in inferring elementary mental organization. New York: Oxford UP.

Mathewson KE, Gratton G, Fabiani M, Beck DM, Ro T (2009) To see or not to see: prestimulus $\alpha$ phase predicts visual awareness. J Neurosci 29:2725-2732.

Montemurro MA, Rasch MJ, Murayama Y, Logothetis NK, Panzeri S (2008) Phase-of-firing coding of natural visual stimuli in primary visual cortex. Curr Biol 18:375-380.

Neggers SF, Raemaekers MA, Lampmann EE, Postma A, Ramsey NF (2005) Cortical and subcortical contributions to saccade latency in the human brain. Eur J Neurosci 21:2853-2863.

O'Hare JJ (1954) The variability of audiory and visual reaction time with change in amplitude and phase of alpha rhythm. Am Psychol 9:444.

Ota T, Toyoshima R, Yamauchi T (1996) Measurements by biphasic changes of the alpha band amplitude as indicators of arousal level. Int J Psychophysiol 24:25-37.

Pastötter B, Hanslmayr S, Bäuml KH (2010) Conflict processing in the anterior cingulate cortex constrains response priming. Neuroimage 50:1599-1605.

Purves D, Paydarfar JA, Andrews TJ (1996) The wagon wheel illusion in movies and reality. Proc Natl Acad Sci U S A 93:3693-3697.

Rajkai C, Lakatos P, Chen CM, Pincze Z, Karmos G, Schroeder CE (2008) Transient cortical excitation at the onset of visual fixation. Cereb Cortex 18:200-209.

Ratcliff R (1978) A theory of memory retrieval. Psychol Rev 85:59-108.

Sato T, Murthy A, Thompson KG, Schall JD (2001) Search efficiency but not response interference affects visual selection in frontal eye field. Neuron 30:583-591

Schall JD, Thompson KG (1999) Neuronal selection and control of visually guided eye movements. Annu Rev Neurosci 22:241-259.

Sirota A, Montgomery S, Fujisawa S, Isomura Y, Zugaro M, Buzsáki G (2008) Entrainment of neocortical neurons and gamma oscillations by the hippocampal theta rhythm. Neuron 60:683-697.

Sparks D, Rohrer WH, Zhang Y (2000) The role of the superior colliculus in saccade initiation: a study of express saccades and the gap effect. Vis Res 40:2763-2777.

Stroud JM (1956) The fine structure of psychological time. Information theory in psychology. Chicago: The Free Press.

Stroud JM (1967) The fine structure of psychological time. Ann NY Acad Sci 138:623-631.

Surwillo WW (1963a) The relation of simple response time to brain-wave frequency and the effects of age. Electroencephalogr Clin Neurophysiol 15:105-114. 
Surwillo WW (1963b) The relation of response-time variability to age and the influence of brain wave frequency. Electroencephalogr Clin Neurophysiol 15:1029-1032.

Surwillo WW (1964) The relation of decision time to brain wave frequency and to age. Electroencephalogr Clin Neurophysiol 16:510-514.

Thut G, Nietzel A, Brandt SA, Pascual-Leone A (2006) Alpha-band electroencephalographic activity over occipital cortex indexes visuospatial attention bias and predicts visual target detection. J Neurosci 26: 9494-9502.

Treisman M, Faulkner A, Naish PL, Brogan D (1990) The internal clock: evidence for a temporal oscillator underlying time perception with some estimates of its characteristic frequency. Perception 19:705743.

van Dijk H, Schoffelen JM, Oostenveld R, Jensen O (2008) Prestimulus os- cillatory activity in the alpha band predicts visual discrimination ability. J Neurosci 28:1816-1823.

VanRullen R, Koch C (2003) Is perception discrete or continuous? Trends Cogn Sci 7:207-213.

Varela FJ, Toro A, John ER, Schwartz EL (1981) Perceptual framing and cortical alpha rhythm. Neuropsychologia 19:675-686.

Venables PH (1960) Periodicities in reaction time. Br J Psychol 51:37-43.

Walsh EG (1952) Visual reaction time and the alpha-rhythm, an investigation of a scanning hypothesis. J Physiol 118:500-508.

White CT, Harter MR (1969) Intermittency in reaction time and perception, and evoked response correlates of image quality. Acta Psychol (Amst) 30:368-377.

Wurtz RH, Albano JE (1980) Visual-motor function of the primate superior colliculus. Annu Rev Neurosci 3:189-226. 